\title{
Interventions
}

\section{The Complexity Behind Face Masks}

\section{Dhaval Adjodah}

Published on: Mar 09, 2021

DOI: $10.21428 / 9610 d d b 2 . c b 961 f 92$

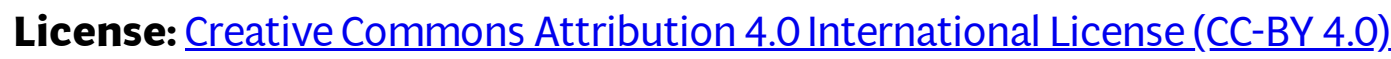




\section{To mask or not to mask?}

Understanding the effect of mask wearing on COVID-19 health outcomes, and what the drivers behind increased mask wearing are critically important questions - at least until we have enough people vaccinated.

Numerous studies have shown that masks have been associated with a reduction in the infection rate among health care workers in a large hospital network, mask mandates have helped reduce the number of cases in the United States and in Germany, and simulations have shown that wearing a mask can protect against droplet infection by preventing the spread of viral particles even when worn imperfectly. Despite this evidence, there has been strong resistance against mask-wearing.

Last week, the states of Texas, Mississippi and Montana lifted their mask mandates, even though these states have some of the lowest vaccination rates in the country. This week, Wyoming lifted its mask mandate. Our results strengthen the case for the adoption of mask mandates and caution against relaxing such recommendations yet especially given that masking is perhaps even beneficial to the economy.

\section{A brief history lesson}

The current complexities and controversies surrounding face masks that have been permeating the scientific, policy and cultural conversations around COVID-19 are not new. You might have seen the old timey pictures of football fans wearing masks during the 1918 pandemic or the one of the Del Perugia family's cat wearing a mask.

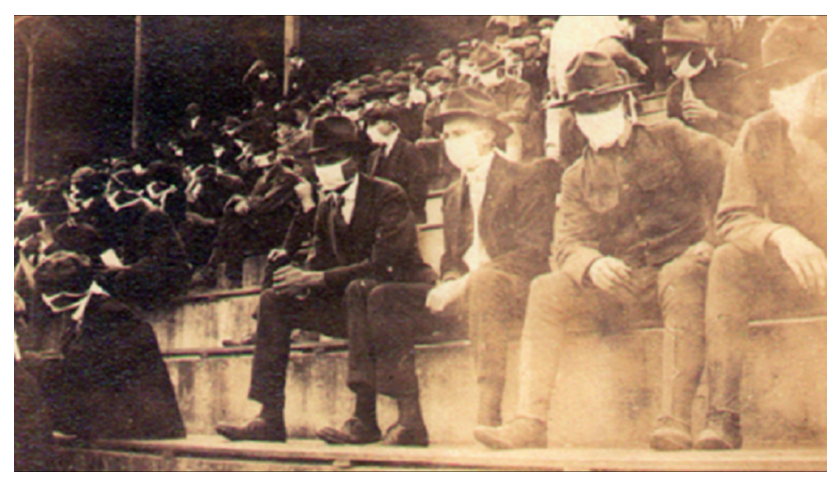




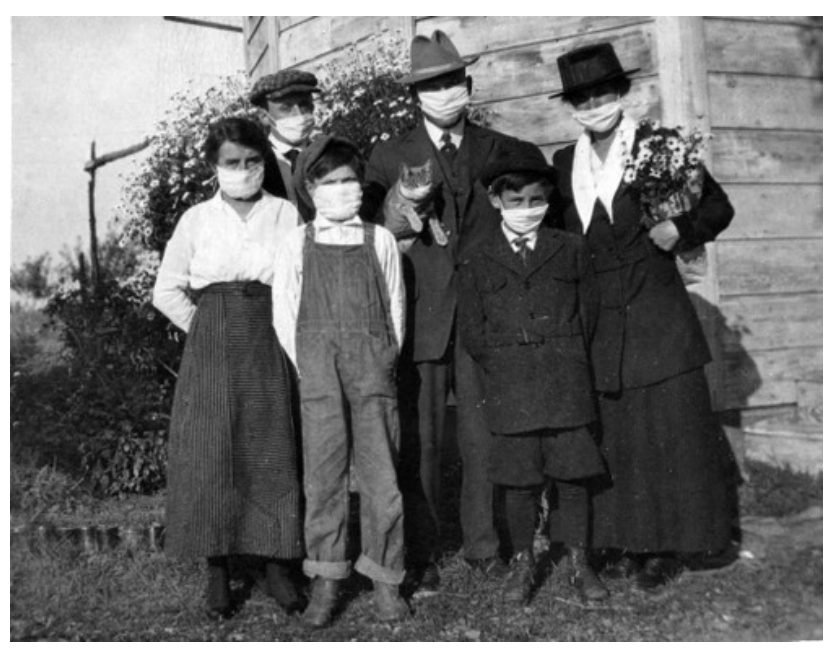

source: top $\underline{\text { bottom }}$

But have you seen the pictures of the arrests and attacks related to the Anti-Mask League of San Francisco in 1918?

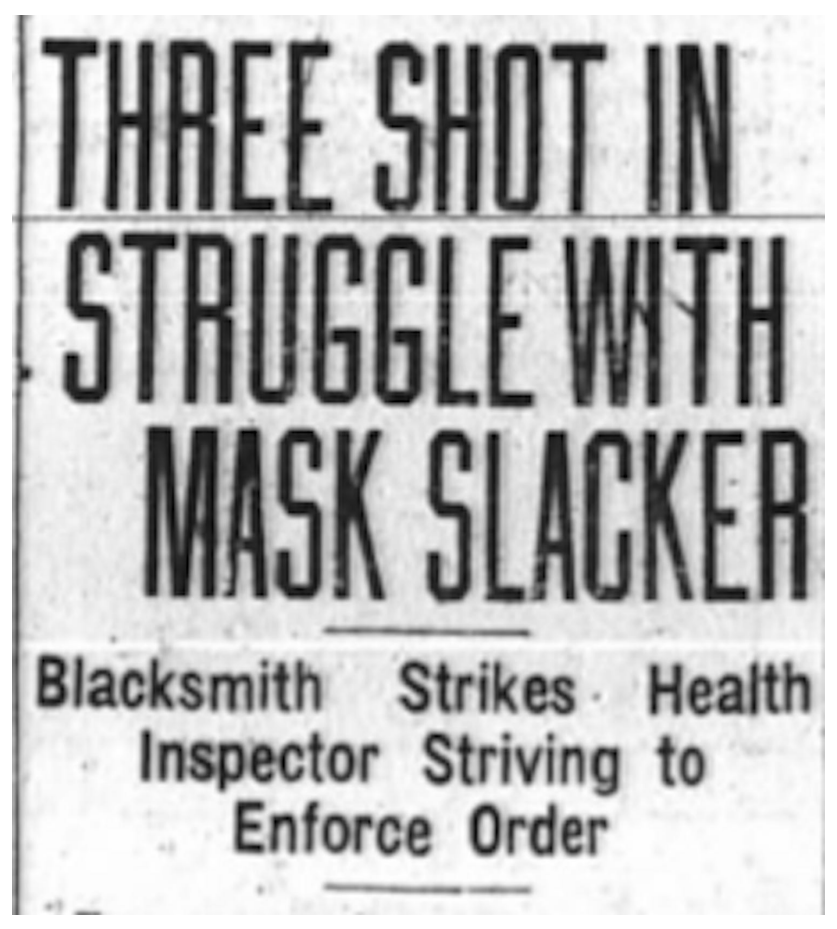




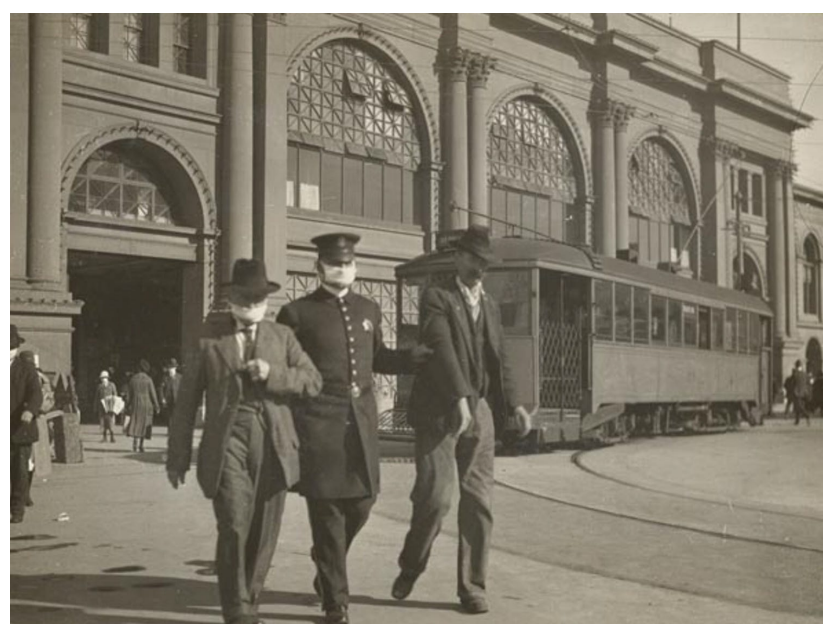

source

History is repeating itself. Not only to we need more evidence of the positive effect of masks, but also we need to understand the effect of norms towards masks.

\section{Our study}

To strengthen the case that masks have a positive effect on the pandemic, we have designed a study (link to paper under peer review here) where we have extended previous studies on the impact of masks on COVID-19 outcomes by investigating an unprecedented breadth and depth of health outcomes, geographical resolutions, types of mask mandates, early versus later waves and controlling for other government interventions, mobility testing rate and weather.

A significant limitation in investigating the effect of masks has been the lack of a consistent dataset capturing all key variables of interest. For example, existing data on mask mandates sometimes disagree due to variations in start date and in the type of mandates being considered. Furthermore, while numerous datasets record daily changes in COVID cases and deaths, they are rarely accompanied by policy information such as the date of introduction and scope of the mask mandates. Additionally, longitudinal data on mask adherence has been missing until recently, even though there are significant ongoing surveys and computer vision-based efforts to estimate mask adherence from social media images and videos.

We therefore undertook the task of unifying disparate public data sources (such as COVID-19 health outcomes such as cases, hospitalization proportions and deaths; controls such as testing rate, weather and mobility; and state and county-level mask mandate dates and scopes) to create a longitudinal dataset that can be used to 
investigate the effect of mask mandates on COVID-19. We share our code and data on Github.

\section{The effect of state and county-level mask mandates on COVID-19 outcomes}

Using the above data, we use the econometrics framework of Event Study designs to estimate treatment effects of mask mandates on COVID-19 health outcomes. We find that mask mandates are associated with a statistically significant decrease in new cases $(-3.55$ per $100 \mathrm{~K})$, deaths $(-0.13$ per $100 \mathrm{~K})$, and the proportion of hospital admissions (-2.38 percentage points) up to 40 days after the introduction of mask mandates both at the state and county level. These effects are large, corresponding to $14 \%$ of the highest recorded number of cases, $13 \%$ of deaths, and $7 \%$ of admission proportion.

This is illustrated in the figure below: mask mandates were introduced at day zero, shown by the red vertical line; the y-axis represents the treatment effect associated with mask mandates on the COVID-19 outcome for each day before or after the introduction of a mask mandate relative to the day before the introduction of a mask mandate. For all three outcomes, we observe that the treatment effect to be negative which means that mask mandates are associated with a decrease in cases, hospitalizations and deaths up to 40 days after the introduction of mask mandates. 


\section{Effect of Mask Mandates on COVID-19 Outcomes}

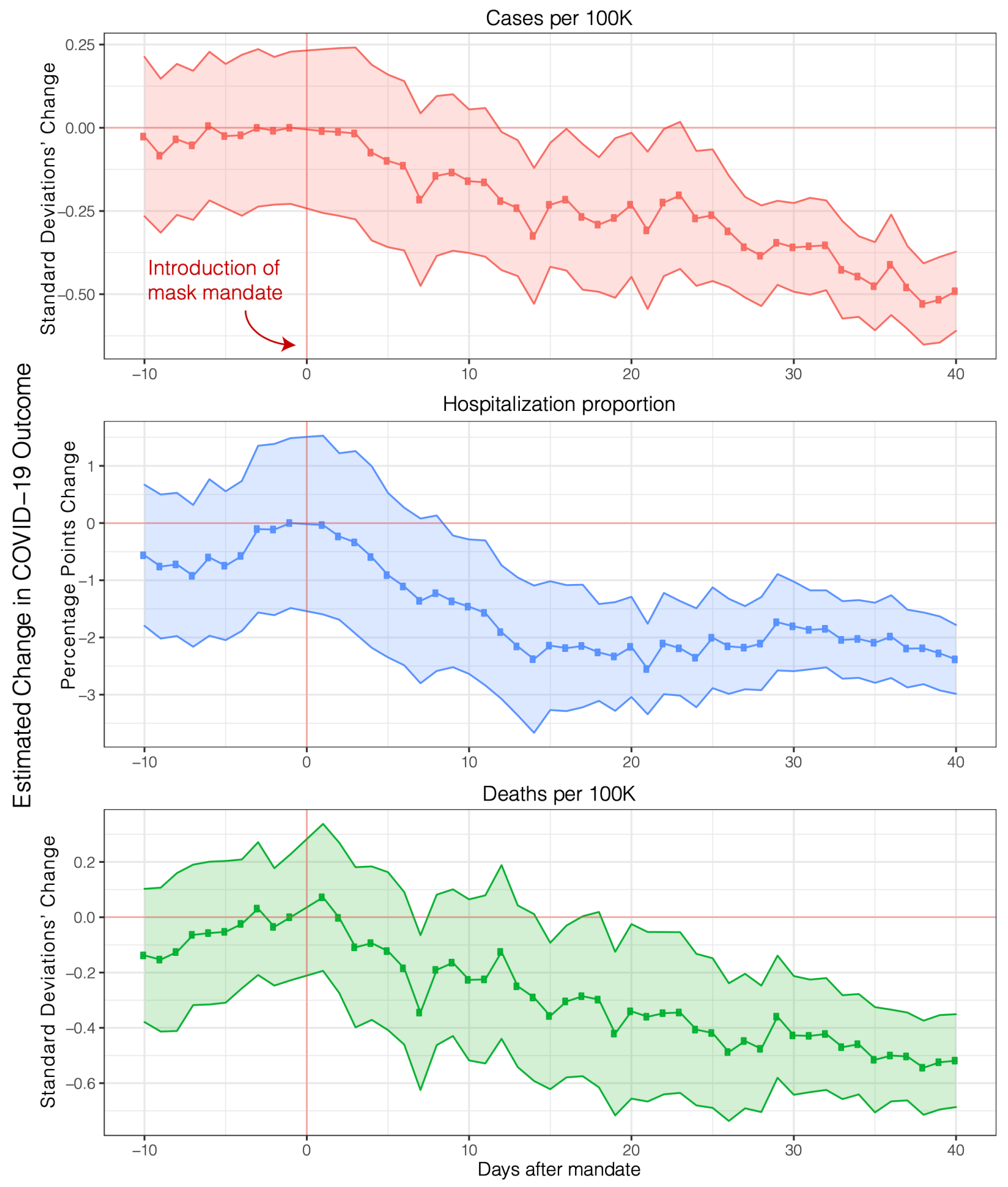

As detailed in the specification of the model below (see paper for the full definitions), we control for many confounders such as mobility, weather, new test rates and other 
Non-Pharmaceutical Interventions (NPIs) i.e. stay-at-home and non-essential business closures.

$C_{o, t, s} \sim \sum_{\gamma=-10}^{40} \gamma_{\tau} \times 1\left(r_{s, t}=\tau\right)+\alpha_{s}+\alpha_{t}+\beta_{s} t+X_{t, s}+C_{o, t-\delta, s}+C_{o, t, s}^{\prime}+\epsilon_{t, s}$

\section{Mask Adherence}

An implicit assumption of previous work on mask mandates is that they increase mask adherence (the proportion of people who wear masks). We put this assumption to the test.

Data that tracks mask usage at a county or state level over time is only now starting to

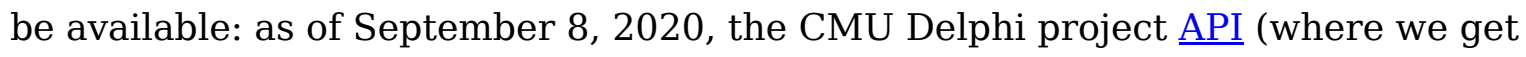
much of our data) has started publicly releasing mask adherence data that measures the estimated percentage of people who wore a mask by asking the question ' 'In the past 5 days, how often did you wear a mask when in public?" in an ongoing Facebook online survey ran on millions of people in the US.

During the period starting September 8 (when adherence data started being available), only 4 states have enacted new state level requirements: Hawaii and Iowa on November 16, North Dakota on November 14 and New Hampshire on November 20.

Using this data, we find that mask mandates are linked to a 23.4 percentage point increase in mask adherence in four diverse states, as in the figure below. We notice a delay of about 4 days before we observe an upward trend in mask adherence which is consistent with behavior change literature. 


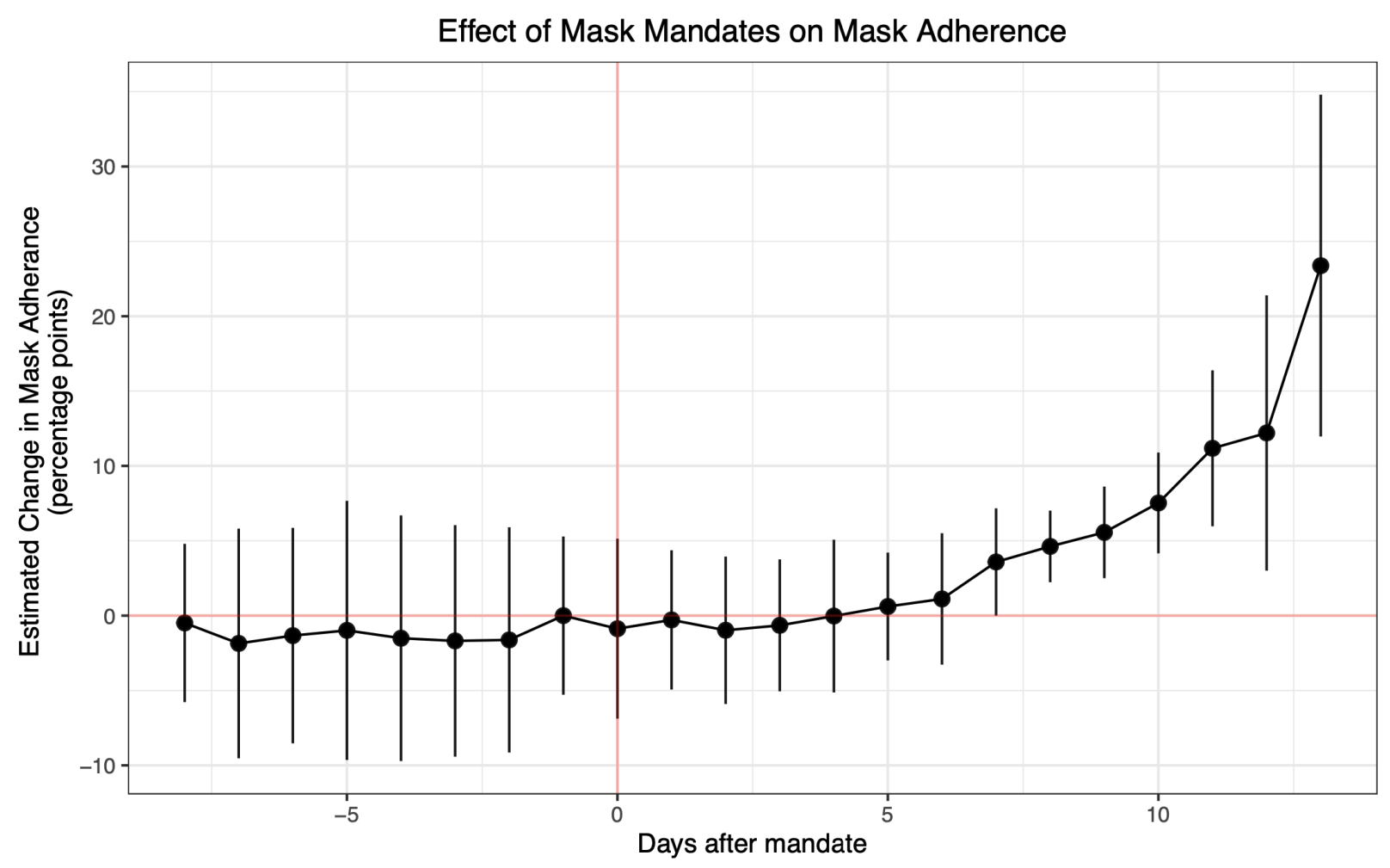

\section{Going international}

So far, we have focused on the US.

We expand the scope of our analysis and look at the effect of mask adherence and attitudes towards mask wearing internationally. We do so by using the novel surveybased dataset COVID-19 Beliefs, Behaviors \& Norms Survey which asks more than 150 questions about COVID-19 to more than half a million online Facebook survey respondents in 51 languages from 68 countries.

We focus on the two questions most relevant to the effect of masks on COVID-19 outcomes: a question about mask adherence: ' Out of 100 people in your community, how many do you think wear a face mask or covering when they go out in public?"; and a question about mask norms: ' Out of 100 people in your community, how many do you think believe the following because of COVID-19: People should wear a face mask or covering when out in public?". The first question is a self-reported sample estimate of the percentage of a respondent's community that wears masks in public, and the second question provides a sample estimate of the percentage of the community that believes masks to be important to wear in public. 
As shown in the figure below, we observe that a 1\% increase in community mask adherence is associated with a decrease of -0.45 cases per $100 \mathrm{~K}$ and a decrease of -0.042 deaths per $100 \mathrm{~K}$. Similarly, we find that a $1 \%$ increase in community attitude about the importance of wearing masks leads to a decrease of -0.53 cases per $100 \mathrm{~K}$ and a decrease of -0.035 deaths per $100 \mathrm{~K}$.

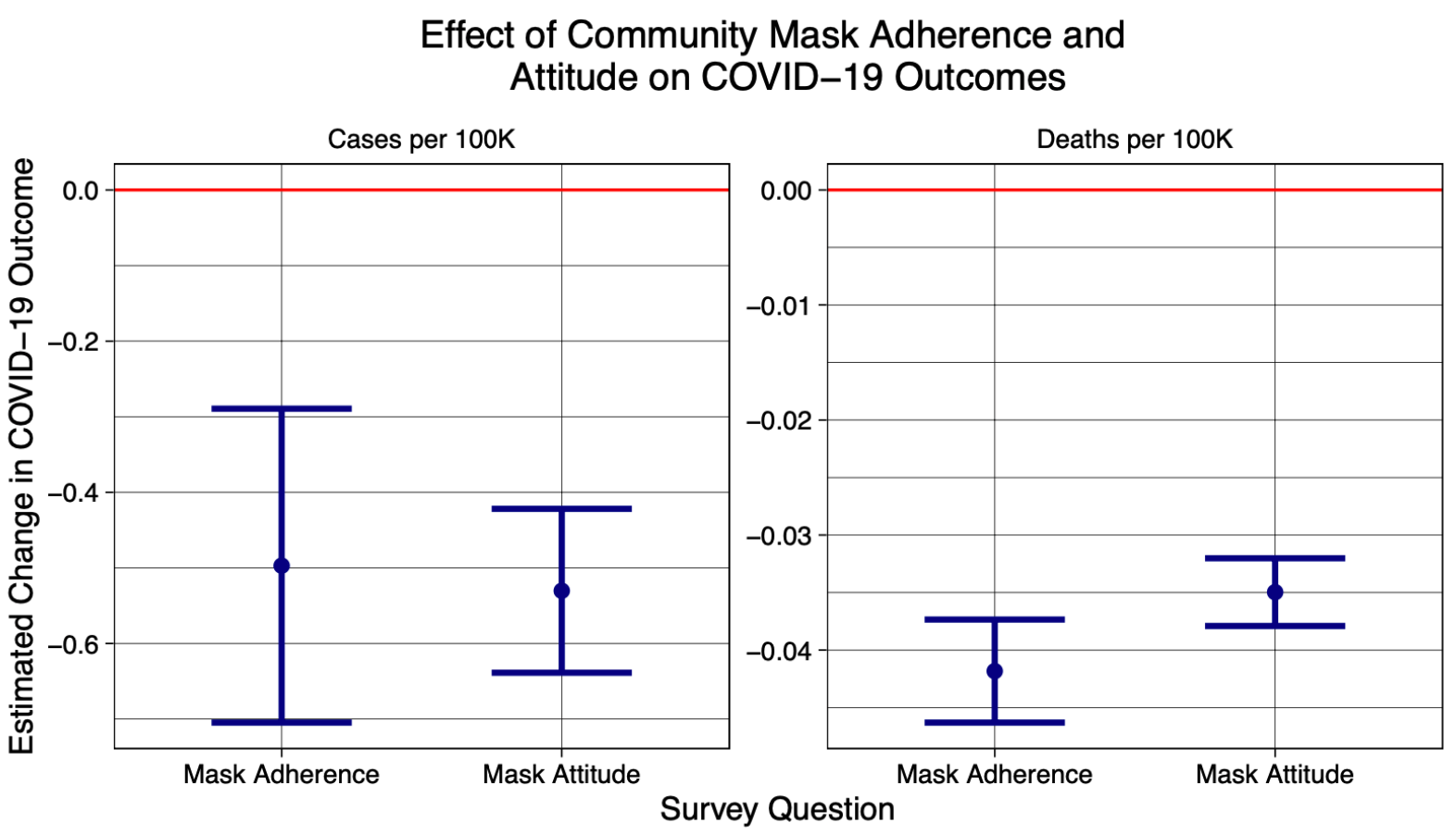

Again, these effects are large: the current statistics in the world as of December 14, 2020 is 10 daily new cases per $100 \mathrm{~K}$ and 0.17 daily deaths per $100 \mathrm{~K}$, which suggest that even a $1 \%$ increase in mask adherence and attitudes is associated with a strong positive impact on COVID-19 outcomes worldwide.

\section{What does it all mean?}

Even though our results, taken together, strongly suggest the positive effect of mask mandates, mask adherence and mask attitudes on COVID-19 cases, hospitalizations and deaths, an important open question is the investigation of the optimal incentives and regulatory mechanisms to increase mask-adherence especially in a climate where conspiracy theories are emerging to discourage mask-adherence.

More research is also needed to probe improvements in public health communication strategies to encourage mask-adherence, including harm-reduction frameworks and policies tailored to help disadvantaged communities' lived experiences. Lessons can be taken from successful approaches in other public health crises such as HIV. 
P.S. paper link \& github $\underline{\text { link }}$ 